\title{
Main limitations of transpulmonary thermodilution: set targets
}

\author{
Manuel Sánchez-Sánchez ${ }^{1,2^{*}}$ (D), Eva Herrero', Lucia Cachafeiro', Eva Flores', Alexander Agrifoglio', \\ Belén Civantos ${ }^{1}$ and Abelardo García-de-Lorenzo ${ }^{1}$ \\ See related Review by Monnet and Teboul https://ccforum.biomedcentral.com/articles/10.1186/s13054-017-1739-5
}

\section{Background}

We read with great interest the review by Xavier Monnet and Jean-Louis Teboul [1]. This review concluded that transpulmonary thermodilution (TPTD) provides a full haemodynamic evaluation. The authors discuss the limitations of previous studies reporting a lack of outcome improvement in patients treated using TPTD-based fluid management. They stated that the objectives of these studies were not comparable and that questionable protocols were used. Based on our previous studies of TPTD in burn patients [2], we would like to make some comments.

The TPTD technique provides parameters that must be interpreted having previous knowledge about situations which may influence results. In addition, the normal values of healthy individuals may not be appropriate comparators for critically ill patients. Therefore, the successful use of this device should be based on the achievement of appropriate objectives [3]. Extravascular lung water or the pulmonary vascular permeability index can be useful parameters for reducing the fluid load if it is elevated, but not to provide more volume if it is low because the patient may not exhibit preload responsiveness or may show only 'temporary' responsiveness. For instance, if there is high capillary leakage, the haemodynamic benefits obtained may be lost in a short time. Furthermore, it is erroneous to equate alteration of the pulmonary vascular permeability index with the systemic vascular permeability index [4]. In any case, this technique can help determine other parameters, such as stroke volume variation, or measure the responses of these parameters to fluid challenge.

In our study, we have found that critically ill patients with slightly low preload values (global end-diastolic volume $<600 \mathrm{ml} / \mathrm{m}^{2}$ ) achieve adequate cardiac index

\footnotetext{
* Correspondence: manuelsanchezsa@gmail.com

${ }^{1}$ Hospital Universitario La Paz-Carlos III/IdiPAZ, Madrid, Spain

${ }^{2}$ Intensive Medicine Service, Hospital Universitario La Paz, Paseo de la Castellana 261, 28046 Madrid, Spain
}

$\left(>2.5 \mathrm{~L} / \mathrm{min} / \mathrm{m}^{2}\right)$ and lactate values $(<2 \mathrm{mmol} / \mathrm{L})$, avoiding excessive volume contributions $[2,5]$.

Finally, it is important to acknowledge possible complications in certain circumstances. For example, in burn patients with severe hypovolemia we found a higher incidence of transient ischaemia in the lower limbs than the $0.4 \%$ reported in some studies.

\section{Conclusions}

We agree with the authors that TPTD provides a full cardiovascular evaluation adequate for most critically ill patients, but we believe that in addition to improving the technique, the user requires adequate knowledge of factors that influence the measurements. We suggest that an adequate cardiac index and tissue perfusion can be achieved with below-normal levels of preload and therefore these values may be adequate in certain situations. We believe that the main limitation of this technique is the lack of accurate targets.

\section{Abbreviation \\ TPTD: transpulmonary thermodilution}

Funding

No funding source.

\section{Availability of data and materials} Not applicable.

\section{Authors' contributions}

MS-S, EH, LC, EF, AA, BC, and AG-d-L drafted the letter. All authors read and approved the final manuscript.

\section{Authors' information \\ Intensive Medicine Service, Hospital Universitario La Paz-Carlos III/IdiPAZ, Paseo de la Castellana 261, Madrid 28046, Spain.}

\section{Ethics approval and consent to participate Not applicable.}

\section{Consent for publication}

Not applicable. 


\section{Competing interests}

The authors declare that they have no conflict of interest.

\section{Publisher's Note}

Springer Nature remains neutral with regard to jurisdictional claims in published maps and institutional affiliations.

Published online: 18 September 2017

\section{References}

1. Monnet $\mathrm{X}$, Teboul JL. Transpulmonary thermodilution: advantages and limits. Crit Care. 2017;21:147.

2. Sánchez SM, García-de-Lorenzo A, Herrero E, López T, Galván B, Asensio MJ, Cachafeiro L, Casado C. A protocol for resuscitation of severe burn patients guided by transpulmonary thermodilution and lactate levels: a 3-year prospective cohort study. Crit Care. 2013;17(4):R176.

3. Monnet X, Marik PE, Teboul JL. Prediction of fluid responsiveness: an update. Ann Intensive Care. 2016;6(1):111.

4. Sánchez $M$, Jiménez-Lendínez $M$, Cidoncha $M$, Asensio MJ, Herrero $E$, Collado A, Santacruz M. Comparison of fluid compartments and fluid responsiveness in septic and non-septic patients. Anaesth Intensive Care. 2011;39(6):1022-9.

5. Sánchez-Sánchez M, García-de-Lorenzo A, Asensio MJ, Herrero E, Cachafeiro L, Agrifoglio A. Effectiveness of treatment based on transpulmonary thermodilution in critically ill patients. Intensive Care Med. 2015;41(6):1154-5. 